\title{
Strong Stability of Perturbed Systems of Volterra Integral Equations
}

\author{
by \\ J. M. Cushing \\ Department of Mathematics \\ University of Arizona \\ Tucson, Arizona 85721
}

Our purpose here is to prove certain stability theorems for the system of Volterra equations

$$
u(t)=u_{0}(t)+\int_{a}^{t}[K(t, s) u(s)+f(t, s, u(s))] d s
$$

for suitable perturbation terms $f, f(t, s, 0) \equiv 0$, under the assumption that the unperturbed, linear system

$$
v(t)=u_{0}(t)+\int_{a}^{t} K(t, s) v(s) d s
$$

has certain stability properties. Here $u, v, u_{0}$ and $f$ are $n$-vectors and $K$ is an $n \times n$ matrix. We assume that $u_{0}(t) \in C\left[t_{0},+\infty\right)$ and that $K(t, s)$ and $f(t, s, z)$ are continuous for $t, s \geq t_{0}$ and $t, s \geq t_{0},|z|<r$, respectively (for some fixed constants $t_{0}>-\infty$ and $\left.0<r \leq+\infty\right)$. This guarantees, for each $a \geq t_{0}$, the local existence and continuability of a continuous solution to (P) (for $|u(a)|<r$ ) and the global existence and uniqueness of the solution to (L) [1, Chap. 2]. Consequently, to each solution of (P) we may associate a maximal interval of existence $\left(t_{m}, t_{M}\right)$ where $t_{0} \leq t_{m}<a<t_{M} \leq+\infty$ and where either $t_{M}=+\infty$ or $\lim _{t \rightarrow t_{M}}|u(t)|=r$ and either $t_{m}=t_{0}$ or $\lim _{t \rightarrow t_{m}}|u(t)|=r$ [1, p. 93].

We deal here with several notions of "strong" stability as introduced in [2] for linear systems (L), all of which are motivated by the concept of strong stability in the theory of ordinary differential equations (see [3] and the references cited therein). In [2] these stabilities were characterized for (L) in terms of boundedness properties of the fundamental matrix $U(t, s)$, defined as the $n \times n$ matrix solution of the matrix equation

$$
U(t, s)=I+\int_{s}^{t} K(t, \tau) U(\tau, s) d \tau, \quad t, s \geq t_{0}
$$

These results together with a representation (or "variation of constants") formula for the solutions of (P) (see (R) below) will lead us to stability results for $(\mathrm{P})$ analogous to those for strong stability of ordinary differential equations [3] and uniform and asymptotic stability for integral equations [4]. 
Let $N$ be a set of "initial functions", $u_{0}(t)$, contained in $C\left[t_{0},+\infty\right)$ and $|\cdot|$ any $n$-vector norm. As in [2] we make the following definition. The system (P) (or, perhaps more exactly, the null solution of (P) corresponding to $u_{0}(t) \equiv 0$ ) is called strongly stable on $N$ if to each $\epsilon>0$ there corresponds a $\delta=\delta(\epsilon)>0$ (independent of $a \geq t_{0}$ ) such that any solution $u(t)$ of $(\mathrm{P})$ for $u_{0}(t) \in N$ satisfying $\left|u\left(t^{*}\right)\right| \leq \delta$ for some $t^{*} \in\left(t_{m}, t_{M}\right)$ necessarily exists and satisfies $|u(t)| \leq \epsilon$ for all $t \geq t_{0}$. In considering $(\mathrm{P})$ we will find that a related, but weaker, concept of stability becomes important: (P) is called ultimately strongly stable on $N$ if there exists a constant $a^{*} \geq t_{0}$ such that to each $\epsilon>0$ there corresponds a $\delta=\delta(\epsilon)>0$ for which any solution $u(t)$ of (P) with $u_{0} \in N$ and $a \geq a^{*}$ satisfying $\left|u\left(t^{*}\right)\right| \leq \delta$ for some $t^{*} \in\left(t_{m}, t_{M}\right)$ necessarily exists and satisfies $|u(t)| \leq \epsilon$ for all $t \geq a^{*}$. Note that the two stabilities are equivalent if and only if $a^{*}=t_{0}$. Below (Remark 2) we will give an example of a (linear) system which is ultimately strongly stable but not strongly stable on $E^{1}$ (Euclidean space). It is clear, however, that a strongly stable system is ultimately strongly stable.

We will also consider two other types of "strong" stabilities as introduced in [2]. Let $N$ have a norm $\|\cdot\|_{N}$. As in [4] equation (P) is stable on $N$ if to $\epsilon>0$ there exists a $\delta=\delta(a, \epsilon)>0$ such that $\left\|u_{0}\right\|_{N} \leq \delta$ implies $u(t)$ exists and satisfies $|u(t)| \leq \epsilon$ for all $t \geq t_{0}$. We say that $(\mathrm{P})$ is adjointly stable on $N$ if it is stable on $N$ and, in addition, to any $\epsilon>0$ and $t \geq t_{0}$ there corresponds a $\delta=\delta(t, \epsilon)>0$ such that $\left\|u_{0}\right\|_{N} \leq \delta$ implies $|u(t)| \leq \epsilon$ for all $a \geq t_{0}$. Finally, (P) is uniformly adjointly stable on $N$ if to each $\epsilon>0$ there exists a $\delta=\delta(\epsilon)>0$ (independent of $a \geq t_{0}$ ) such that $\left\|u_{0}\right\|_{N} \leq \delta$ implies $u(t)$ exists and satisfies $|\dot{u}(t)| \leq \epsilon$ for all $t \geq t_{0}$.

Strong stability clearly implies uniform adjoint stability whereas the converse is false [2]. Also it is clear that uniform adjoint stability implies adjoint stability; the converse, however, remains an open question (but is conjectured to be false). Inasmuch as ultimate strong stability says nothing about (P) for $a \geq a^{*}$, it, in general, neither implies nor is implied by adjoint or uniform adjoint stability.

Our goal is to find what stability properties of (L) will imply a given stability property of $(\mathrm{P})$ when the perturbation term $f$ satisfies the following (DiniHukuhara type) condition:

(H) Assume that $f(t, s, z)$ and $f_{t}(t, s, z)$ are continuous for $t, s \geq t_{0}$ and $|z|<r$ and that there exist non-negative functions $\gamma_{1}(t), \gamma_{2}(t, s)$ for $t, s \geq t_{0}$ such that for all $|z|<r$

$$
\begin{gathered}
|f(t, t, z)| \leq \gamma_{1}(t)|z|, \quad t \geq t_{0} ; \\
\left|f_{t}(t, s, z)\right| \leq \gamma_{2}(t, s)|z|, \quad t \text { and } s \geq t_{0} ; \\
\text { where } \gamma^{*}=\int_{t_{0}}^{+\infty} \gamma(t) d t<+\infty, \gamma(t)=\gamma_{1}(t)+\int_{t_{0}}^{t} \gamma_{2}(t, s) d s .
\end{gathered}
$$

If $f$ is independent of $t$, then $(\mathrm{H})$ is a well-known condition in the stability theory of ordinary differential equations [3]. In [4] the condition $(\mathrm{H})$ is used to study the uniform and asymptotic stability of $(P)$.

Before stating and proving our first theorem, we make a few observations concerning the strong stability of (L) on a linear space $N$. Define $R=\left\{u_{0}\left(t_{0}\right)\right.$ : $\left.u_{0}(t) \in N\right\} \subseteq E^{n}$. Clearly the map $L: N \rightarrow R$ defined by $L\left[u_{0}\right]=u_{0}\left(t_{0}\right)$ is linear. 
Moreover, $L$ is one-to-one; for suppose that $u_{0}\left(t_{0}\right)=u_{0}^{*}\left(t_{0}\right)$ for $u_{0}, u_{0}^{*} \in N$, $u_{0}(t) \neq u_{0}^{*}(t)$. Then, denoting the solution of $(\mathrm{L})$ by $v\left(t ; a, u_{0}\right)$, we have by the linearity of (L) that $v\left(t ; a, u_{0}-u_{0}^{*}\right)=v\left(t ; a, u_{0}\right)-v\left(t ; a, u_{0}^{*}\right)$ and hence $v\left(t_{0} ; a\right.$, $\left.u_{0}-u_{0}^{*}\right)=0$. But the strong stability of (L) on $N$ and the fact that $u_{0}-u_{0}^{*} \in N$ then imply that $v\left(t ; a, u_{0}-u_{0}^{*}\right)=0$ for all $t \geq t_{0}$ because of the non-oscillatory property of strongly stable systems (see [2]). This in turn implies $u_{0}(t) \equiv u_{0}^{*}(t)$, a contradiction. Thus, we have the interesting fact that $N$ can be put into oneto-one correspondence with a linear subspace of $E^{n}$. (Consequently, if $E^{n} \subseteq N$ it follows that $E^{n}=N$ so that $E^{n}$ is in this sense a maximal linear space on which (L) can be strongly stable.) For $t, a \geq t_{0}$, define the linear map $P(t, a): R \rightarrow R(t, a)=\left\{v\left(t ; a, u_{0}\right): u_{0} \in N\right\}$ by $P(t, a)[r]=v\left(t ; a, L^{-1} r\right), r \in R$. Using again the non-oscillatory property of $(\mathrm{L})$, we can show that $P(t, a)$ is invertible for all $t, a \geq t_{0}$. Specifically, if $P(\bar{t}, \bar{a})[\bar{r}]=0$ for some $\bar{t}, \bar{a} \geq t_{0}$ and $\bar{r} \in R$, then $v\left(t ; \bar{a}, L^{-1} \bar{r}\right) \equiv 0$ for $t \geq t_{0}$ which implies $L^{-1} \bar{r}=0$ or $\bar{r}=0$. Now $v\left(t ; a, u_{0}\right)=P(t, a)\left[u_{\mathrm{c}}\right]=P(t, a) P^{-1}(s, a) P(s, a)\left[u_{0}\right]=P(t, a) P^{-1}(s, a)$ $\left[v\left(s ; a, u_{0}\right)\right]$ for all $t, s, a \geq t_{0}$ and $u_{0} \in N$. Referring to the definition of strong stability we find that it is equivalent to the uniform continuity (in $t, s, a \geq t_{0}$ ) of the linear operator $P(t, a) P^{-1}(s, a)$. Thus, there exists a constant $K>0$ independent of $t, a$, and $s$ such that

$$
\left|P(t, a) P^{-1}(s, a)[r]\right| \leq K|r| \quad \text { for } t, s, a \geq t_{0} \text { and } r \in R(s, a) .
$$

This bound is a slight generalization of the characterization of strong stability of (L) on $N=E^{n}$ as proved in [2] since in this special case $P(t, a) \equiv U(t, a)$.

THEOREM 1. Suppose that $f$ satisfies $(H)$, that $(L)$ is uniformly adjointly stable on $E^{n}$, and that $(L)$ is strongly stable on some linear space $N$. Then $(a)(P)$ is ultimately strongly stable on $N$ and $(b)$ there exists a constant $\gamma_{0}, 0<\gamma_{0}<+\infty$, such that if $\gamma^{*}<\gamma_{0}$ then $(P)$ is strongly stable on $N$.

Remarks. (1) If $N=E^{n}$ then because strong stability implies uniform adjoint stability for (L), the assumption in Theorem 1 that $(\mathrm{L})$ is uniformly adjointly stable on $E^{n}$ is superfluous.

(2) The conclusions (a) and (b) of the theorem offer a trade-off between the smallness of $\gamma^{*}$ and the strength of the resulting strong stability of (P). In general, under the hypotheses of the theorem, one cannot conclude the fullfledged strong stability of (P) on $N$ unless $\gamma^{*}$ is in fact small enough, as in part (b). This is illustrated by the following scalar example $(n=1)$. Define $K(t, s)$ $\equiv 0, t$ and $s \geq t_{0}=0$, for which (L) is surely strongly stable on $N=E^{1}$ (since $\left.v(t) \equiv u_{0} \in E^{1}\right)$. Let $f(t, s, z) \equiv p(t, s) z$, where $p(t, s) \equiv s-t$ on $S(\pi)=$ $\{(t, s): 0 \leq t \leq \pi, 0 \leq s \leq \pi\} ; p(t, s) \equiv 0$ for $t \geq 2 \pi ; p(t, s) \equiv 0$ for $s \geq 2 \pi$; and $p(t, s)$ is defined smoothly on $S(2 \pi)-S(\pi)$ (say, so that $p(t, s)$ and $p_{t}(t, s)$ are continuous). Then $|f(t, t, z)| \leq \gamma_{1}(t)|z|$ with $\gamma_{1}(t) \equiv p(t, t)$ and $\left|f_{t}(t, s, z)\right|$ $\leq \gamma_{2}(t, s)|z|$ with $\gamma_{2}(t, s) \equiv p_{t}(t, s)$ for $t, s \geq 0$ and all $z \in E^{1}$. Moreover, $\gamma^{*}=$ $\int_{0}^{2 \pi} p(t, t) d t+\int_{0}^{2 \pi} \int_{0}^{t} p_{t}(t, s) d s d t<+\infty$ and hence $f$ indeed satisfies $(\mathrm{H})$ so that all the hypotheses of Theorem 1 are fulfilled. Equation (P), however, is not strongly stable on $E^{1}$. This can be seen by letting $u_{0}(t) \equiv 1$ and $a=0$, for which (P) has the solution $u(t)=\cos t$ for $0 \leq t \leq \pi$ which, because $u(\pi / 2)=0$, implies $(\mathrm{P})$ is not non-oscillatory on $E^{1}$ and hence not strongly stable on $E^{1}$. 
The system (P) is, as asserted by the theorem, ultimately strongly stable on $E^{1}$ however. One can simply take $a^{*}=2 \pi$ and note that for $t, a \geq a^{*}$ the equation (P) reduces to the trivial (and strongly stable) equation $u(t)=u_{0} \in E^{1}$.

(3) If $N=E^{1}$ and both $K$ and $f$ are independent of $t$, then (L) and (P) are equivalent to a system of ordinary differential equations and its perturbation. Theorem 1 represents an extension of a theorem on strong stability of Coppel $[3, p .66]$ to the more general case of Volterra integral equations, but does not, strictly speaking, offer a generalization of this theorem in that for differential equations the assumption that $\gamma^{*}$ must be small is not needed for the strong stability of (P). Coppel's theorem is included, however, in Theorem 2 below, which deals with uniform adjoint stability on (P).

Proof of Theorem 1. Fundamental to our method is the following representation formula for the solution of $(\mathrm{P})$ :

$$
u(t)=v(t)+\int_{a}^{t} U(t, s) \frac{d}{d s} \int_{a}^{s} f(s, \tau, u(\tau)) d \tau d s,
$$

which is valid on $\left(t_{m}, t_{M}\right)$ for all $a \geq t_{0}$ and $u_{0}(t) \in C\left[t_{0},+\infty\right)$ (see [4]). By (1), we know that $|v(t)| \leq K\left|v\left(t^{*}\right)\right|$ for all $t, t^{*} \geq t_{0}$ and all $a \geq t_{0}, u_{0}(t) \in N$. Also, the assumed uniform adjoint stability of (L) on $E^{n}$ implies the existence of a constant $L>0$ (independent of $t, s \geq t_{0}$ ) for which $|U(t, s)| \leq L, t$ and $s \geq t_{0}$ ([2], Theorem 4). (We use the usual matrix norm: $|U|=\sup _{|r|=1}|U r|$.)

Choose $a^{*} \geq t_{0}$ so large that $\gamma_{a^{*}} \exp \left(\gamma_{a^{*}}\right)<(L K)^{-1}, \gamma_{a^{*}}=\int_{a^{*}}^{+\infty} \gamma(s) d s$. Let $a \geq a^{*}$. From (R), with $t$ replaced by $t^{*}$, we have (using (H)) for all $t^{*} \in$ $\left[a, t_{M}\right)$,

$$
\begin{aligned}
\left|v\left(t^{*}\right)\right| & \leq\left|u\left(t^{*}\right)\right|+L \int_{a}^{t^{*}}\left[\gamma_{1}(s)|u(s)|+\int_{a}^{s} \gamma_{2}(s, \tau)|u(\tau)| d \tau\right] d s \\
& \leq\left|u\left(t^{*}\right)\right|+L \int_{a}^{t^{*}} \gamma(s) p(s) d s
\end{aligned}
$$

where $p(t)=\sup _{[a, t]}|\mathcal{u}(s)|$. Also from (R) for $t \in\left[a, t_{M}\right)$ we have

$$
|u(t)| \leq K\left|v\left(t^{*}\right)\right|+L \int_{a}^{t} \gamma(s) p(s) d s,
$$

or, taking the supremum over $[a, t]$,

$$
0 \leq p(t) \leq K\left|v\left(t^{*}\right)\right|+L \int_{a}^{t} \gamma(s) p(s) d s .
$$

This inequality is valid for $t, t^{*} \in\left[a, t_{M}\right)$ and hence, using Gronwall's theorem, we find $0 \leq p(t) \leq K^{\prime}\left|v\left(t^{*}\right)\right|$ for all $t, t^{*} \in\left[a, t_{M}\right)$. From (2) follows

$$
0 \leq p(t) \leq K^{\prime}\left[\left|u\left(t^{*}\right)\right|+L \int_{a}^{t^{*}} \gamma(s) p(s) d s\right],
$$

for all $t, t^{*} \in\left[a, t_{M}\right)$. The right-hand side of (3) represents a bound on $p(t)$ independent of $t$ which may be used to estimate the integral appearing in (3). This leads to

$$
0 \leq p(t) \leq K^{\prime}\left[\left|u\left(t^{*}\right)\right|+L \gamma_{a} K^{\prime}\left[\left|u\left(t^{*}\right)\right|+L \int_{a}^{t^{*}} \gamma(s) p(s) d s\right]\right] .
$$


The integral here may again be bounded by using (3). Repetition of this procedure leads to the estimate

$$
0 \leq p(t) \leq K^{\prime}\left|u\left(t^{*}\right)\right|\left(1+L \gamma_{a} K^{\prime}+\left(L \gamma_{a} K^{\prime}\right)^{2}+\cdots+\left(L \gamma_{a} K^{\prime}\right)^{n}+\cdots\right),
$$

which (because $a \geq a^{*}$ implies $\gamma_{a} \leq \gamma_{a^{*}}$ ) implies, by the way in which $a^{*}$ was chosen, that

$$
0 \leq|u(t)| \leq p(t) \leq K^{\prime \prime}\left|u\left(t^{*}\right)\right|
$$

for all $t, t^{*} \in\left[a, t_{M}\right)$, where $K^{\prime \prime}=K^{\prime} /\left(1-L \gamma_{a^{*}} K^{\prime}\right)$ is independent of $a \geq a^{*}$.

Inequality (4) can also be shown to be valid for $t, t^{*} \in\left(t_{m}, a\right]$ (with possibly a different $K^{\prime \prime}$, but we let $K^{\prime \prime}$ in (4) be the larger of the two constants) by reflecting the variable $t$ in (P) through $t=a$ and repeating verbatim the argument above. We omit these details and simply conclude that (4) is valid for all $t, t^{*} \in\left(t_{m}, t_{M}\right)$ and $u_{0}(t) \in N$ for a constant $K^{\prime \prime}$ independent of $t, t^{*}$ and $a \geq a^{*}$. Now, for $\epsilon>0$ let $\delta=\delta(\epsilon)=\min \left(r / 2 K^{\prime \prime}, \epsilon / K^{\prime \prime}\right)$. Then if $\left|u\left(t^{*}\right)\right| \leq \delta$ for some $t^{*} \in\left(t_{m}, t_{M}\right)$, it follows from (4) that $|u(t)| \leq r / 2<r$ for all $t \in\left(t_{m}, t_{M}\right)$. But, as mentioned above, either $u(t)$ exists for all $t \geq a^{*}$ or $|u(t)| \rightarrow r$ as $t \rightarrow t_{m}$ or $t \rightarrow t_{M}$. Consequently, if $\left|u\left(t^{*}\right)\right| \leq \delta$ for some $t^{*} \in\left(t_{m}, t_{M}\right)$, then $u(t)$ exists for all $t \geq a^{*}$ (i.e., $t_{M}=+\infty$ and $t_{m}=a^{*}$ ) and inequality (4) is valid for all $t \geq a^{*}$ and $u_{0}(t) \in N$. Thus, by the way $\delta$ was chosen, if $\left|u\left(t^{*}\right)\right| \leq \delta$ for some $t^{*} \geq a^{*}$, then $|u(t)| \leq \epsilon$ for all $t \geq a^{*}$; that is, (P) is ultimately strongly stable on $N$ and part (a) is proved.

Clearly if $\gamma^{*} \exp \left(\gamma^{*}\right)<(L K)^{-1}$ then we can take $a^{*}=t_{0}$ above. This proves (b) and completes the proof of the theorem.

We now turn to consider adjoint and uniform adjoint stability for (P). As can be seen in Theorem 2 and its proof below, it is not difficult to establish that uniform adjoint stability is preserved from (L) to $(P)$ under condition $(H)$. As it is well known, both in the theory of differential equations and integral equations [4], that, with enough uniformity in $a$, stability is preserved under perturbation, this result is not surprising. Since uniformity in $a$ to some extent is also necessary for the preservation of stability, it is not clear to what extent, if any, adjoint stability will be preserved under perturbation, especially in view of the fact that the relationships amongst uniform, adjoint, and uniform adjoint stabilities are not all known [2]. Nonetheless, we can offer a theorem for the adjoint stability of $(\mathrm{P})$ at least when $(\mathrm{P})$ is known already to be stable (Theorem 3).

THEOREM 2. Suppose that $f$ satisfies $(H)$ and that $(L)$ is uniformly adjointly stable on $E^{n}$. Then the uniform adjoint stability of $(L)$ on any set $N$ implies the uniform adjoint stability of $(P)$ on $N$.

We call a set $N$ a cone if $u_{0}(t) \in N$ implies $k u_{0}(t) \in N$ for all constants $k$.

THEOREM 3. Suppose that $f$ satisfies $(H)$ but with the condition $\gamma^{*}<+\infty$ replaced by the assumption that $\gamma(t)$ is integrable on every finite subinterval of $\left[t_{0},+\infty\right)$. Suppose also that $(L)$ is uniformly adjointly stable on $E^{n}$. If $N$ is a cone on which $(L)$ is adjointly stable and $(P)$ is stable, then $(P)$ is adjointly stable on $N$. 
Proof of Theorem 2. By assumption, to each $\epsilon>0$ there corresponds a $\delta^{\prime}=\delta^{\prime}(\epsilon)>0$ such that $\left\|u_{0}\right\|_{N} \leq \delta^{\prime}, u_{0} \in N$, implies $|v(t)| \leq \epsilon$ for all $t \geq t_{0}$. Given $\epsilon>0$, choose $\delta=\delta(\epsilon)=\min \left(\delta^{\prime}\left(\epsilon \exp \left(-L \gamma^{*}\right)\right), \delta^{\prime}\left((1 / 2) r \exp \left(-L \gamma^{*}\right)\right)\right)$ $>0$. Here $L>0$ is a constant such that $|U(t, s)| \leq L$ for all $t, s \geq t_{0}$, which exists by virtue of the assumed uniform adjoint stability of $(\mathrm{L})$ on $E^{n}$ [2]. For $t \in\left[a, t_{M}\right)$ we have from $(\mathrm{R})$ the estimate

$$
|u(t)| \leq|v(t)|+L \int_{a}^{t} \gamma(s) p(s) d s,
$$

where $p(t)=\sup _{[a, t]}|u(s)|$. If $\left\|u_{0}\right\|_{N} \leq \delta$, then by the choice of $\delta$ we have

$$
|u(t)| \leq(1 / 2) r \exp \left(-L \gamma^{*}\right)+L \int_{a}^{t} \gamma(s) p(s) d s,
$$

which, after the supremum over $[a, t]$ has been taken and Gronwall's theorem has been applied to the resulting inequality for $p(t)$, leads to $|u(t)| \leq p(t)<r / 2$ $<r$ for $t \in\left[a, t_{M}\right)$. Once again, as in the proof of Theorem 1 , the continuability property of solutions to (P) implies that $u(t)$ exists for all $t \geq a$. As in the proof of Theorem 1, a reflection of $t$ through $a$ and a repetition of the above argument shows that $u(t)$ exists for all $t \geq t_{0}$ and hence that (5) is valid for all $t \geq t_{0}$. Knowing this, we see that $\left\|u_{0}\right\|_{N} \leq \delta$ implies, inasmuch as $\delta \leq \delta^{\prime}$ $\left(\epsilon \exp \left(-L \gamma^{*}\right)\right)$, that

$$
|u(t)| \leq \epsilon \exp \left(-L \gamma^{*}\right)+L \int_{a}^{t} \gamma(s) p(s) d s
$$

for $t \geq a$. This leads again to $|u(t)| \leq p(t) \leq \epsilon$ for $t \geq a$. Once again a reflection of $t$ through $a$ and a repeated argument as above yields $|u(t)| \leq \epsilon$ for all $t \geq t_{0}$, $u_{0} \in N$, and hence $(\mathrm{P})$ is uniformly adjointly stable on $N$. This proves Theorem 2 .

Proof of Theorem 3. The adjoint stability of (L) on $E^{n}$ implies $|U(t, s)| \leq$ $L(s)$ for $t \geq t_{0}$ and $|U(t, s)| \leq L(t)$ for $s \geq t_{0}$ for some function $L(\cdot)>0$ [2]. Denoting the solution of $(\mathrm{L})$ by $v\left(t ; a, u_{0}\right)$, we find from the adjoint stability of (L) on the cone $N$ that for fixed $t \geq t_{0}$ and each $\epsilon>0$ there exists a $\delta^{\prime}=\delta^{\prime}(t, \epsilon)$ $>0$ such that $\left|v\left(t ; a, \delta^{\prime} u_{0} /\left\|u_{0}\right\|_{N}\right)\right| \leq \epsilon$ for all $a \geq t_{0}$ and $u_{0} \in N\left(\right.$ since $\delta^{\prime} u_{0} /\left\|u_{0}\right\|_{N}$ $\epsilon N$ and $\left.\left\|\delta^{\prime} u_{0} /\right\| u_{0}\left\|_{N}\right\|_{N} \leq \delta^{\prime}\right)$. Fix $\epsilon=\epsilon_{0}>0$. By the linearity of (L), $v(t ; a$, $\left.\delta^{\prime} u_{0} /\left\|u_{0}\right\|_{N}\right)=\delta^{\prime} v\left(t ; a, u_{0}\right) /\left\|u_{0}\right\|_{N}$ and hence $\left|v\left(t ; a, u_{0}\right)\right| \leq K(t)\left\|u_{0}\right\|_{N}$ for all $a \geq t_{0}$ and $u_{0} \in N$, where $K(t)=\epsilon_{0} / \delta^{\prime}\left(t, \epsilon_{0}\right)$.

Since (P) is assumed to be stable on $N$, we only need to show that for each $t \geq t_{0}$ and $\epsilon>0$ there exists a $\delta=\delta(t, \epsilon)>0$ such that $\left\|u_{0}\right\|_{N} \leq \delta, u_{0} \in N$, implies $|u(t)| \leq \epsilon$ for all $a \geq t_{0}$. To this end, set $\delta=\delta(t, \epsilon)=\epsilon / M^{\prime}(t)$, where $M^{\prime}(t)=M(t) \exp \left(\int_{t_{0}}^{t} B(s) d s\right), B(s)=\gamma_{1}(s) M(s)+\int_{t_{0}}^{s} \gamma_{2}(s, \tau) M(\tau) d \tau, M(s)=$ $\max (K(s), L(s))>0$. It is not difficult to see that $\delta^{\prime}(t, \epsilon)>0$ may always be taken as continuous in its arguments and hence that $K(t)$ is continuous in $t \geq t_{0}$. Inasmuch as $L(t)$ is constructed in the same manner as $K(t)$ (see [2], or take $N=E^{n}$ above), it can also be taken as continuous in $t \geq t_{0}$. This, together with the hypothesis on $f$ in Theorem 3, ensures the existence of $M^{\prime}(t)>0$ (and hence $\delta$ ) for each $t \geq t_{0}$. 
From (R) we find, for $t \geq a$, that:

$$
\begin{aligned}
|u(t)| & \leq K(t)\left\|u_{0}\right\|_{N}+L(t) \int_{a}^{t}\left(\gamma_{1}(s)|u(s)|+\int_{a}^{s} \gamma_{2}(s, \tau)|u(\tau)| d \tau\right) d s \\
& \leq M(t)\left[\left\|u_{0}\right\|_{N}+\int_{a}^{t} B(s) p(s) d s\right]
\end{aligned}
$$

where $p(t)=\sup _{[a, t]}|u(s) / M(s)|>0$. Dividing both sides of this inequality by $M(t)>0$, taking the supremum over $[a, t]$ of both sides, and finally applying Gronwall's theorem to the resulting inequality for $p(t)$, we arrive at

$$
0 \leq p(t) \leq\left\|u_{0}\right\|_{N} \exp \left(\int_{t_{0}}^{t} B(s) d s\right) \text {. }
$$

Once again, by reflecting $t$ through $a$ and repeating the argument, we can assert that this estimate is valid for all $t \geq t_{0}$. Thus, since $|u(t)| \leq M(t) p(t)$, we have $|u(t)| \leq\left\|u_{0}\right\|_{N} M(t) \exp \left(\int_{t_{0}}^{t} B(s) d s\right) \leq \epsilon$ for all $a \geq t_{0}$ provided $\left\|u_{0}\right\|_{N}$ $\leq \delta, u_{0} \in N$. This proves Theorem 3 .

All theorems above presuppose knowledge of the stability properties of the linear system $(\mathrm{L})$. With respect to this problem we point out the results and techniques presented in [5] and [6].

\section{REFERENCES}

[1] R. K. Muler, Nonlinear Volterra Integral Equations, W. A. Benjamin, Menlo Park, California, 1971.

[2] J. M. Bownds and J. M. Cushing, On strong stability for linear integral equations, Math. Systems Theory 7 (1973), 193-200.

[3] W. A. CoppeL, Stability and Asymptotic Behavior of Differential Equations, D. C. Heath and Co., Boston, 1965.

[4] J. M. Bownds and J. M. CusHING, Some stability theorems for systems of Volterra integral equations, J. Appl. Anal., to appear.

[5] J. M. Bownds and J. M. CushING, Some stability criteria for linear systems of Volterra integral equations, Funkcial. Ekvac. 15 (1972), 101-117.

[6] J. M. Bownds and J. M. CusHING, On stability for Volterra integral equations using an associated differential system, IBM Research Report No. RC 3750, T. J. Watson Research Center, Yorktown Heights, N.Y., 10598.

(Received 24 October 1972) 